ISSN 2310-2861

www.mdpi.com/journal/gels

Article

\title{
Composite Chitosan/Agarose Ferrogels for Potential Applications in Magnetic Hyperthermia
}

\section{Vanessa Zamora-Mora ${ }^{1}$, Paula I.P. Soares ${ }^{2}$, Coro Echeverria ${ }^{2}$, Rebeca Hernández ${ }^{1, *}$ and Carmen Mijangos ${ }^{1, *}$}

1 Instituto de Ciencia y Tecnología de Polímeros (ICTP-CSIC) c/ Juan de la Cierva 3, 28006 Madrid, Spain; E-Mail: vzamora4@gmail.com

2 CENIMAT/I3N, Departamento de Ciência dos Materiais, Faculdade de Ciências e Tecnologia, FCT, Universidade Nova de Lisboa, 2829-516 Caparica, Portugal;

E-Mails: paulaipsoares@gmail.com (P.I.P.S.),coro@fct.unl.pt (C.E.)

* Author to whom correspondence should be addressed; E-Mails: rhernandez@ictp.csic.es (R.H.); cmijangos@ictp.csic.es (C.M.); Tel.: +34-915-6229-00 (ext. 271) (C.M.)

Academic Editor: Rolando Barbucci

Received: 12 May 2015 / Accepted: 3 July 2015 / Published: 9 July 2015

\begin{abstract}
Composite ferrogels were obtained by encapsulation of magnetic nanoparticles at two different concentrations (2.0 and $5.0 \% \mathrm{w} / \mathrm{v})$ within mixed agarose/chitosan hydrogels having different concentrations of agarose $(1.0,1.5$ and $2.0 \%(w / v))$ and a fixed concentration of chitosan $(0.5 \%(w / v))$. The morphological characterization carried out by scanning electron microscopy showed that dried composite ferrogels present pore sizes in the micrometer range. Thermogravimetric measurements showed that ferrogels present higher degradation temperatures than blank chitosan/agarose hydrogels without magnetic nanoparticles. In addition, measurements of the elastic moduli of the composite ferrogels evidenced that the presence of magnetic nanoparticles in the starting aqueous solutions prevents to some extent the agarose gelation achieved by simply cooling chitosan/agarose aqueous solutions. Finally, it is shown that composite chitosan/agarose ferrogels are able to heat in response to the application of an alternating magnetic field so that they can be considered as potential biomaterials to be employed in magnetic hyperthermia treatments.
\end{abstract}

Keywords: chitosan; agarose; magnetic nanoparticles; magnetic hyperthermia 


\section{Introduction}

Hydrogels are defined as three-dimensional polymer networks that are able to retain a large amount of water in their swollen state [1]. Specifically, hydrogels obtained from natural polymers are currently the focus of considerable scientific research for the development of biomedical applications due to their inherent biocompatibility, biodegradability and also because they are susceptible to enzymatic degradation [2-4]. An example of natural polymer commonly employed for the preparation of hydrogels is chitosan. Chitosan is soluble in certain acidic aqueous solutions due to the protonation of the primary amine groups. Due to the $\mathrm{pH}$-responsiveness and inherent biocompatibility, chitosan gels are attractive for biomedical applications [5-7] and specially, as materials for controlled drug delivery [8-11].

Chitosan hydrogels can be obtained either by physical associations, such as secondary forces (hydrogen, ionic, or hydrophobic bonding) and physical entanglements and by covalent crosslinking [2]. Chitosan aqueous solutions can become a gel in alkaline solutions without any chemical cross-linkers due to the fact that positive charges of chitosan molecules are neutralized resulting in coacervation-phase inversion with gel formation. This property can be employed for the in situ synthesis of iron oxide nanoparticles employing chitosan gels as templates. In a first step, iron cations are solubilized in an acid aqueous solution of chitosan and, as a second step, the oxidation in alkaline solution leads to the simultaneous formation of magnetic nanoparticles (magnetite or maghemite) and chitosan gelation [12]. Another method to prepare magnetic chitosan hydrogels consists in the encapsulation of preformed magnetic nanoparticles into a chitosan matrix [13,14]. These hybrid chitosan hydrogels, also known as ferrogels, can be implemented as materials for biomedical applications, i.e., materials employed in magnetic hyperthermia, that is, able to heat up target tumors remotely through an external magnetic field [15-17].

In order to broaden the applications of chitosan hydrogels, researchers have developed composite hydrogels, where chitosan is blended with other biopolymers, e.g. collagen [18], gelatin [19] and agarose [20]. In particular, agarose, an alternating copolymer found in some seaweeds consisting of 1,4-linked 3,6-anhydro- $\alpha$-l-galactose and 1,3-linked $\beta$-d-galactose derivatives is a neutral polysaccharide that forms thermoreversible gels upon cooling agarose aqueous solutions. The biocompatibility of agarose hydrogels and the mild conditions required for its gelation, make the composite hydrogel suitable for biomedical applications, in particular, for cell culture and sustained drug delivery [21]. In addition, mixing of agarose with chitosan can be used to improve the mechanical properties of agarose, [22,23] or properties of adhesion of cells [23]. Recently, mixtures of chitosan and agarose have been prepared by a suspension cross-linking method [24], water-in-oil emulsion technique [25] or via electrospinning [26].

The gelation of agarose in mixed aqueous solutions of chitosan and agarose leads to the formation of transparent and mechanically stable hydrogels in which chitosan chains remain embedded into the agarose matrix. In this way, the $\mathrm{pH}$ and temperature response of the resulting composite gels is retained [27]. The aim of this work is to collective evidence of a facile and simple approach to obtain biodegradable and biocompatible ferrogels from natural sources and their ability to respond to an applied external magnetic field, as an indication of the feasibility to be employed as potential materials for magnetic hyperthermia. In this work, we employ the same methodology previously used to obtain Chitosan/Agarose (Chi/Aga) composite ferrogels, without chemical crosslinking, through 
encapsulation of magnetic nanoparticles dispersed as an aqueous ferrofluid at two different concentrations and we report the preparation method, the morphology, the mechanical properties as a function of temperature, and the Specific Power Absorption (SPA) measurements.

\section{Results and Discussion}

\subsection{Morphological Study}

Figure 1 shows the macroscopic appearance of a composite Chi/Aga-1.5 hydrogel and its corresponding ferrogels loaded with two different concentrations of ferrofluid $(2.0 \%$ and $5.0 \%(w / v))$. Chi/Aga blank hydrogels are transparent in the hydrated state, which suggest uniform distribution of chitosan in the host agarose matrix, without noticeable phase separation, as previously reported [27]. On the other hand, Chi/Aga ferrogels $(\mathrm{Chi} / \mathrm{Aga}+\mathrm{Fe})$ present the characteristic brown color corresponding to the ferrofluid, which gives evidence to the encapsulation of magnetic nanoparticles within the polymer hydrogels.

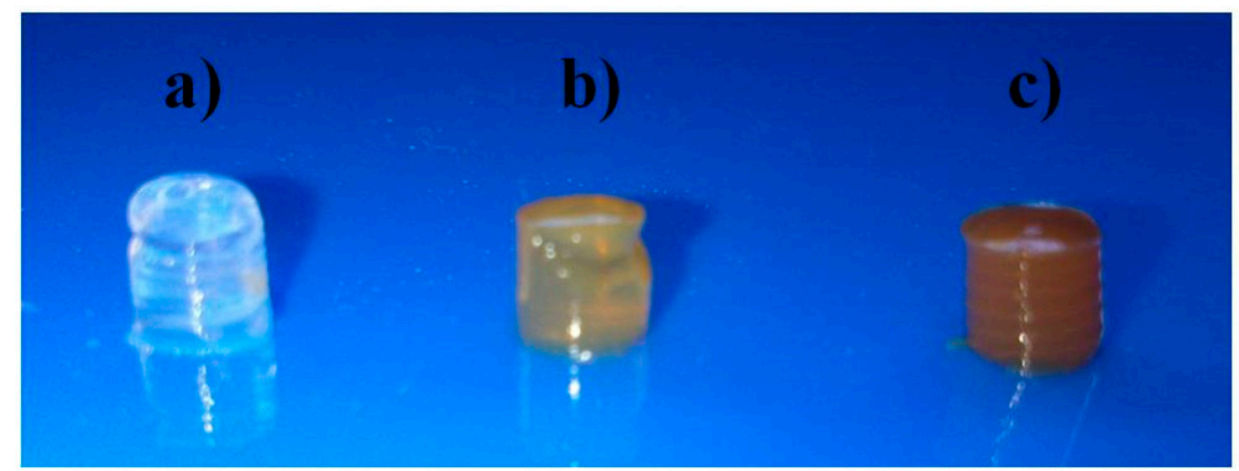

Figure 1. Photographs of (a) Chi/Aga-1.5 hydrogel and its ferrogels (b) $\mathrm{Chi} /$ Aga- $1.5+\mathrm{Fe} 2 \%$ and (c) $\mathrm{Chi} /$ Aga- $1.5+\mathrm{Fe} 5 \%$.

The morphology of the dried ferrogels, Chi/Aga-1.5+Fe2\% and Chi/Aga-1.5+Fe5\% was further analyzed through SEM. The results corresponding to the surface and cross section are shown in Figure 2. As can be observed, both samples present a rough surface. The pore size determined from the images corresponding to the cross section was $37 \pm 5 \mu \mathrm{m}$ for Chi/Aga- $1.5+\mathrm{Fe} 2 \%$ and $51 \pm 9 \mu \mathrm{m}$ for Chi/Aga-1.5+Fe5\%. 
a) Surface

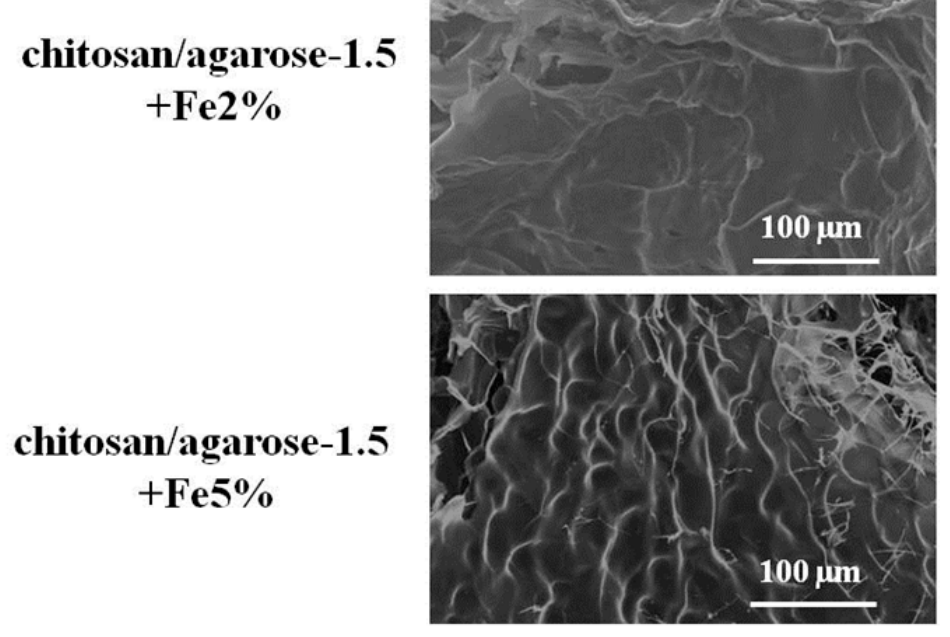

b) Cross section
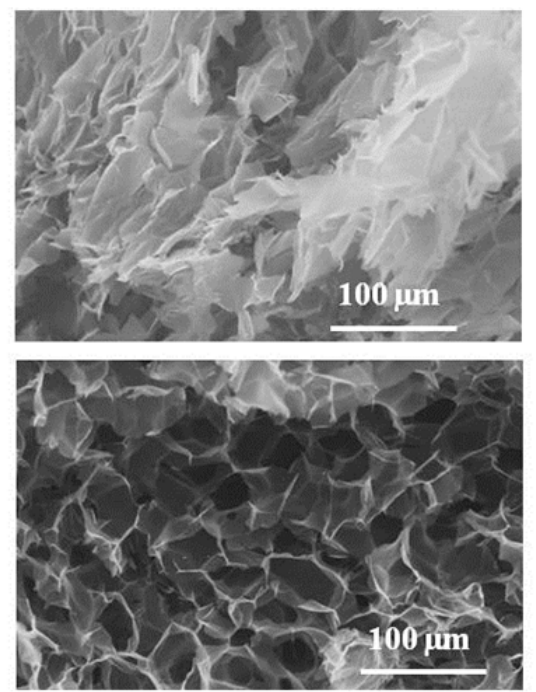

Figure 2. SEM images corresponding to the (a) surface and (b) cross section of Chi/Aga-1.5 ferrogels loaded with magnetic nanoparticles at $2.0 \%$ and $5.0 \%(w / v)$.

\subsection{Thermal Stability}

The thermal stability of dried composite Chi/Aga hydrogels and ferrogels was determined through thermogravimetric analysis. Representative results corresponding to samples Chi/Aga-2, $\mathrm{Chi} / \mathrm{Aga}-2+\mathrm{Fe} 2 \%$ and $\mathrm{Chi} / \mathrm{Aga}+\mathrm{Fe} 5 \%$ are shown in Figure 3. The three samples presented a first weight loss $(\sim 9 \%)$ between 25 and $80{ }^{\circ} \mathrm{C}$, which is attributed to the evaporation of superficial water. Then, the second weight loss stage in the range of $150-500{ }^{\circ} \mathrm{C}$ is due to the decomposition of the organic chains of polymers, chitosan [28] and agarose [29] with similar weight losses, $\sim 75 \%$. According to the derivative of TGA (Figure 3b), the corresponding degradation temperatures for Chi/Aga-2, Chi/Aga-2+Fe2\% and Chi/Aga-2+Fe5\% are 294, 307 and $308{ }^{\circ} \mathrm{C}$, respectively. The difference among degradations temperatures of composite ferrogels could be attributed to the establishment of interactions among the polymer matrix and the magnetic nanoparticles, as previously reported [12] for other nanocomposite polymer materials.

The residues obtained for Chi/Aga-2, Chi/Aga-2+Fe2\% and Chi/Aga-2+Fe $5 \%$ at $600{ }^{\circ} \mathrm{C}$ are $12 \%$, $15 \%$ and $16 \%$, respectively. The magnetite content $\left(\mathrm{Fe}_{3} \mathrm{O}_{4} \% w / v\right)$ in composite chitosan/agarose ferrogels can be determined by subtracting the residue corresponding to the blank hydrogel (Chit/Aga-2), being 3 and 4\% (w/w) for Chi/Aga-2+Fe2\% and Chi/Aga-2+Fe5\%, respectively. 

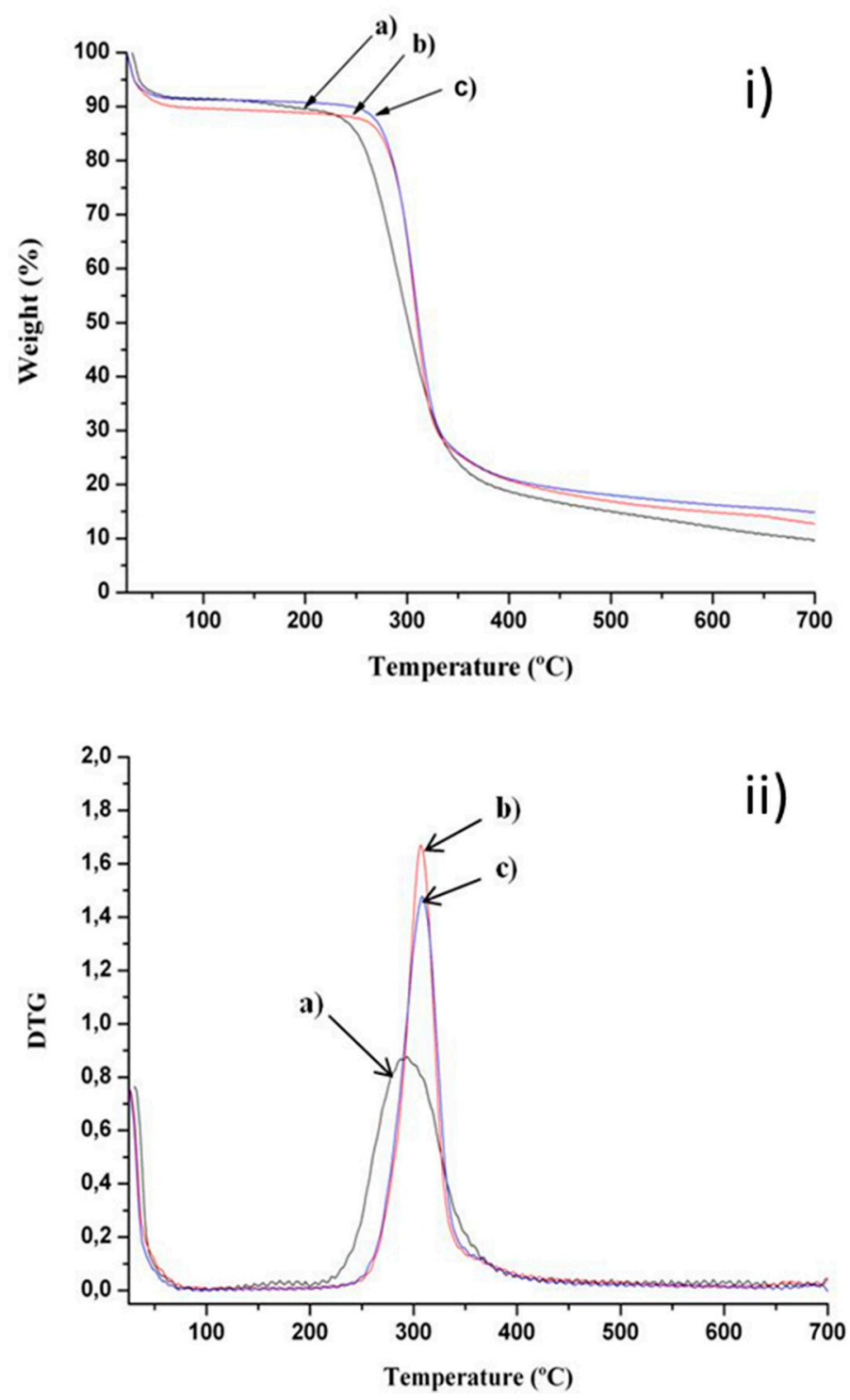

Figure 3 (i) Thermal degradation and (ii) differential thermogravimetric curves of (a) Chi/Aga-2 (black), (b) Chi/Aga-2+Fe2\% (red) and (c) Chi/Aga-2+Fe $\%$ (blue).

\subsection{Viscoelastic Properties}

Figure 4 shows the variation in elastic modulus, $\mathrm{G}^{\prime}$, with temperature of Chi/Aga ferrogels loaded with the same concentration of ferrofluid $(5 \%(w / v))$ at different concentrations of agarose. As can be observed, the elastic modulus increases with the agarose content and although not shown here, $\mathrm{G}^{\prime}>\mathrm{G}^{\prime \prime}$ at temperatures lower than the melting temperature of the gel, as previously reported for Chi/Aga hydrogels [27].

The melting temperature $\left(T_{\mathrm{m}}\right)$ of $\mathrm{Chi} / \mathrm{Aga}-1+\mathrm{Fe} 5 \%$, Chi/Aga- $1.5+\mathrm{Fe} 5 \%$ and $\mathrm{Chi} / \mathrm{Aga}-2+\mathrm{Fe} 5 \%$ ferrogels is $48.2 \pm 0.9{ }^{\circ} \mathrm{C}, 48.4 \pm 0.6{ }^{\circ} \mathrm{C}$ and $49.5 \pm 0.9{ }^{\circ} \mathrm{C}$, respectively. These $T_{\mathrm{m}}$ values are lower than those previously reported for blank composite Chi/Aga hydrogels, which was $57 \pm 1{ }^{\circ} \mathrm{C}$ for Chi/Aga-1 and $62 \pm 1{ }^{\circ} \mathrm{C}$ for Chi/Aga-2 hydrogels [27]. This might be attributed to a lower degree of crosslinking of the agarose in the presence of ferrofluid, which might indicate that the encapsulation of magnetic nanoparticles within composite Chi/Aga hydrogels prevents to some extent the agarose gelation. This is reinforced by the fact that the $\mathrm{G}^{\prime}$ values obtained at temperatures below $T_{\mathrm{m}}$ are lower 
for Chi/Aga ferrogels than those corresponding to blank Chi/Aga hydrogels as can be observed in the inset of Figure 4.

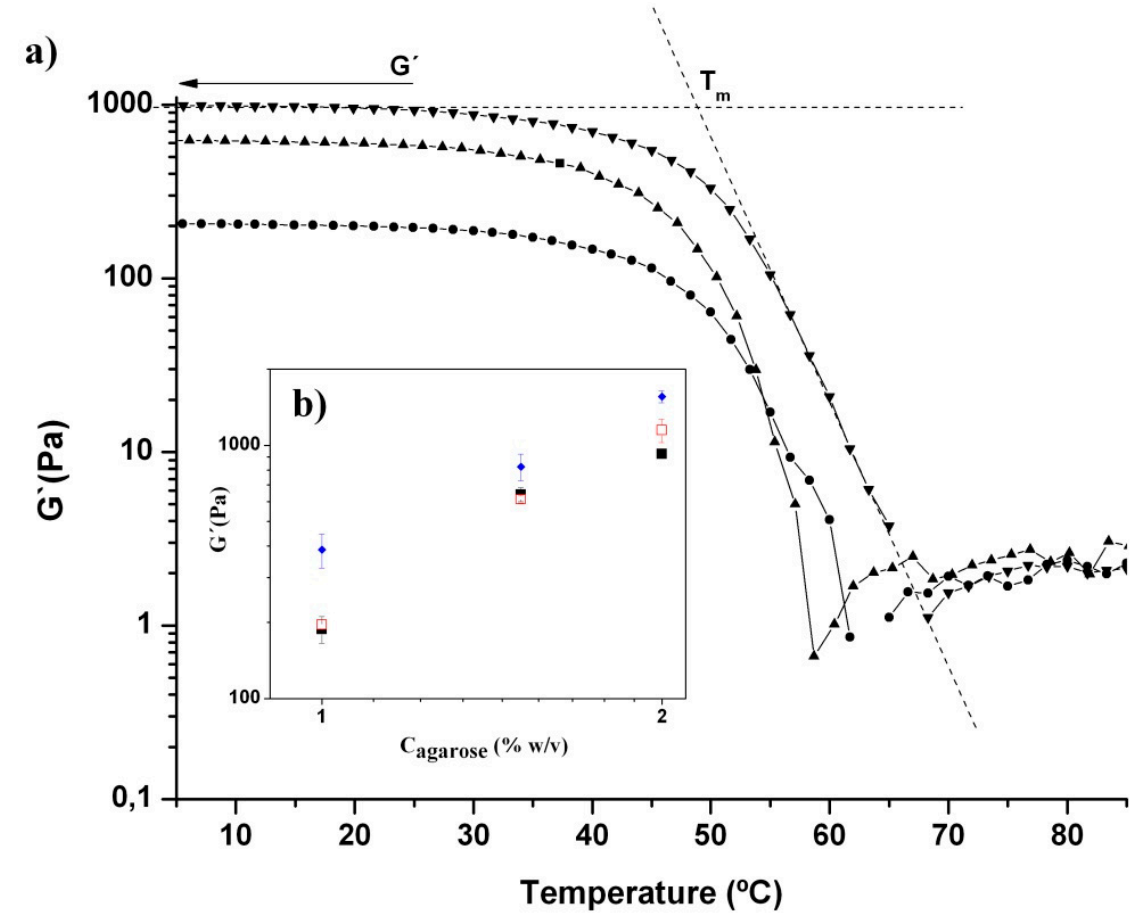

Figure 4. (a) Elastic modulus, $\mathrm{G}^{\prime}$, plotted as a function of temperature for (•) Chi/Aga-1+Fe5\%, ( $\mathbf{\Delta}$ ) Chi/Aga-1.5+Fe $5 \%$ and ( $)$ ) Chi/Aga-2+Fe5\%. Dashed lines show the determination of the melting temperature, $T_{\mathrm{m}}$, of the gel. (b) Inset image corresponds to the variation in $\mathrm{G}^{\prime}$ as a function of agarose concentration for Chi/Aga ferrogels loaded with

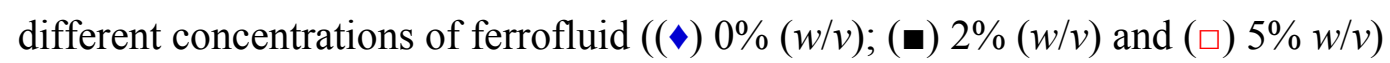

\subsection{Specific Power Absorption Experiments}

Figure 5 shows the heating performance of Chi/Aga-1.5 loaded with two concentrations of ferrofluid $(2 \%$ and $5 \%(w / v))$, when the samples are submitted to an alternating magnetic field (AMF). As can be observed, there is an increase of temperature with time as a function of the ferrofluid content, being a temperature increase of $(\Delta T) \sim 2.5{ }^{\circ} \mathrm{C}$ for $\mathrm{Chi} / \mathrm{Aga}-1.5+\mathrm{Fe} 5 \%$ and $\sim 1.5{ }^{\circ} \mathrm{C}$ for Chi/Aga-1.5+Fe2\% after having been subjected to an alternating magnetic field for $900 \mathrm{~s}$. 


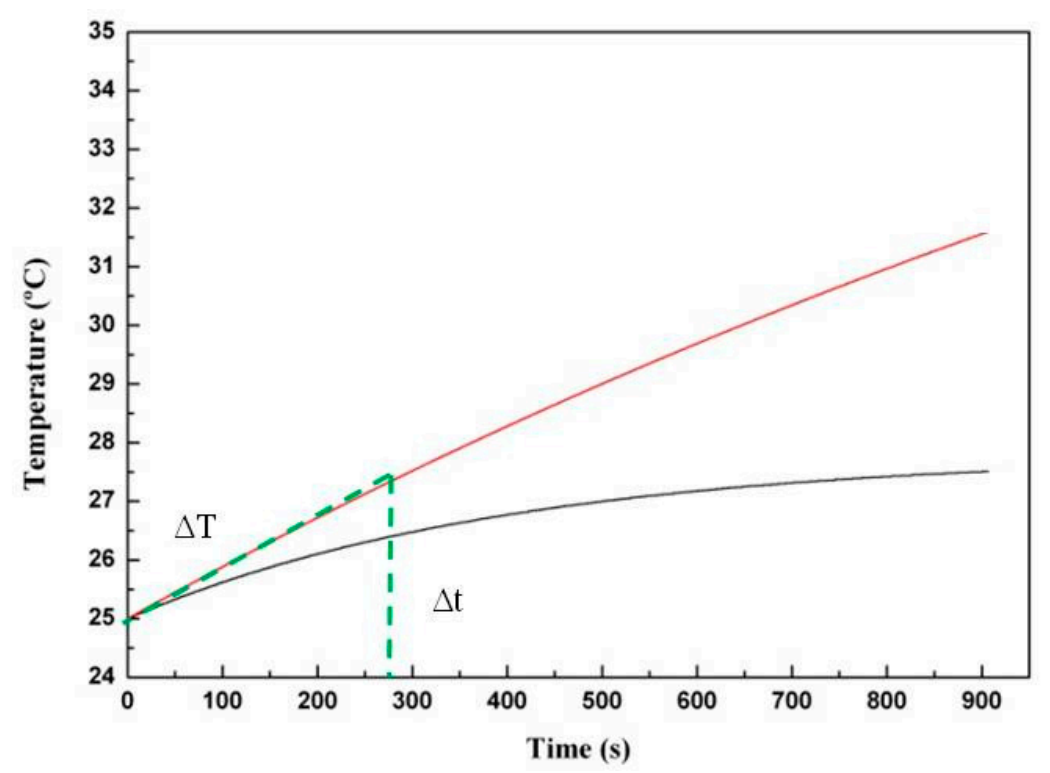

Figure 5. Representative heating performance of Chi/Aga-1.5 ferrogels loaded with ferrofluid $(2 \%(w / v)$ (black line) and 5\% (w/v) (red line)), measured at $f=418.5 \mathrm{kHz}$ and $24 \mathrm{kA} / \mathrm{m}$.

Specific Power Absorption (SPA) measures the capacity of heat generation of Chi/Aga ferrogels when submitted to an alternating magnetic field. The SPA of the ferrogels was determined from the initial slope of the temperature vs. time curves (green part of Figure 5). The temperature increase $(\Delta T)$ of a given mass of ferrofluid diluted in the system corresponds to that of the selected time interval $(\Delta t)$, in our case $275 \mathrm{~s}$. SPA was calculated by the following equation [30]:

$$
S P A=\frac{m_{L I Q} c_{L I Q}+m_{N P} c_{N P}}{m_{N P}}\left(\frac{\Delta T}{\Delta t}\right)
$$

where $\mathrm{C}_{\mathrm{LIQ}}$ and $\mathrm{c}_{\mathrm{NP}}$ are the specific heat capacities of the liquid carrier and the nanoparticles, respectively. Due to the low concentration of the magnetic material in the gels the following approximation $m_{L I Q} c_{L I Q}+m_{N P} c_{N P} \approx m_{L I Q} c_{L I Q}$ can be applied, so that the equation to calculate SPA becomes:

$$
S P A=\frac{m_{L I Q} c_{L I Q}}{m_{N P}}\left(\frac{\Delta T}{\Delta t}\right)
$$

The SPA values listed in Table 1 show similar values of SPA for both ferrogels. The differences in the slopes $(\Delta T / \Delta t)$ corresponding to the ferrofluid and the ferrogels are attributed to the low amount of magnetic material that is present in the ferrogels. The results corresponding to SPA are normalized to the concentration of magnetic material so that the differences found with respect to the ferrofluid might be due to differences in the agglomeration of the magnetic material in the ferrogels, which might induce a decrease in their SPA [31,32]. 
Table 1. Specific power absorption of the samples under study.

\begin{tabular}{ccccc}
\hline Samples & $\begin{array}{c}\Delta \boldsymbol{T} / \Delta \boldsymbol{t} \\
\left({ }^{\circ} \mathbf{C} / \mathbf{s}\right)\end{array}$ & $\begin{array}{c}\text { Water content } \\
(\mathbf{\%})\end{array}$ & $\begin{array}{c}\mathbf{F e}_{3} \mathbf{O}_{\mathbf{4}} \\
(\mathbf{m g} / \mathbf{m L})\end{array}$ & SPA (W/g) \\
\hline Ferrofluid & 1.61 & 89.7 & 69.9 & 96 \\
Chi/Aga-1.5+ Fe2\% & 0.007 & 96.8 & 0.96 & 30 \\
Chi/Aga-1.5+ Fe5\% & 0.009 & 97.1 & 1.16 & 32 \\
\hline
\end{tabular}

* For the ferrofluid, $\mathrm{Fe}_{3} \mathrm{O}_{4}(\mathrm{mg} / \mathrm{mL})$ is calculated through UV-vis. In the gels, $\mathrm{Fe}_{3} \mathrm{O}_{4}(\mathrm{mg} / \mathrm{mL})$ was calculated from the residue values obtained from TGA (Figure 3) considering the water content of the samples and the volume of the cell in the magnetic hyperthermia equipment $(1 \mathrm{~mL})$.

\section{Conclusions}

We report on a simple method to obtain composite ferrogels from the combination of two natural polymers, chitosan and agarose. Agarose gelation in the presence of a mixed aqueous solution containing a dispersion of magnetic nanoparticles and chitosan allows the encapsulation of magnetic nanoparticles within the composite matrixes. The resulting ferrogels present a porous structure in the micrometer range and an increased thermal stability with respect to the corresponding blank hydrogels. This might be ascribed to the presence of interactions between the magnetic nanoparticles and the polymer composite matrix. Notwithstanding, a decrease in the elastic moduli and melting temperature of the ferrogels is observed with respect to blank hydrogels, which suggests that the encapsulation of magnetic nanoparticles within the $\mathrm{Chi} /$ Aga hydrogels prevents to some extent agarose gelation. The application of an alternating magnetic field to Chi/Aga ferrogels leads to an increase of temperature so that these materials could be considered potential candidates to be employed in magnetic hyperthermia treatment, even if the low values of SPA obtained with respect to the SPA value corresponding to the initial ferrofluid suggests agglomeration of the magnetic nanoparticles within the polymer composite matrixes.

\section{Experimental Section}

\subsection{Materials}

Chitosan (Chi) employed in this work was isolated from shrimp shell (Heterocarpus vicarious) and supplied by Polymers Laboratory, National University, Costa Rica. Chitosan has a deacetylation degree of $65 \%$ as determined by potentiometric titration and a molecular weight of $362 \mathrm{kDa}$ as determined by viscosity method (ASTM D 2857). Ultra-low gelling temperature agarose (Aga) (gelling temperature in the range of $8-17{ }^{\circ} \mathrm{C}$ and melting point in the range of $40-50{ }^{\circ} \mathrm{C}$ ) was purchased from SeaPrep (Lonza, Switzerland, www.lonza.com). Oleic-acid-coated iron oxide nanoparticles dispersed in water as a ferrofluid (density $=1.08 \mathrm{~g} / \mathrm{mL}$ ), NGAP FeO-05\#4, were provided by Nanogap Subnmparticles, Spain. According to the manufacturer, the crystalline form is magnetite, $\mathrm{Fe}_{3} \mathrm{O}_{4}$ and the average size of nanoparticles is $18.55 \pm 2 \mathrm{~nm}$. The solid content was $10.30 \% \mathrm{w} / \mathrm{w}$. The magnetite concentration in the ferrofluid $\left(\mathrm{Fe}_{3} \mathrm{O}_{4}(\mathrm{mg} / \mathrm{mL})\right)$, was determined through UV spectroscopy. The ferrofluid was digested in $\mathrm{HNO}_{3}$ and $\mathrm{HCl} 6 \mathrm{M}$ and iron concentration was measured spectrophotometrically at the $\lambda_{\max }$ of $478 \mathrm{~nm}$. 


\subsection{Preparation of Composite Chi/Aga Blank Hydrogels and Chi/Aga Ferrogels}

For the preparation of composite Chi/Aga blank hydrogels, different amounts of agarose were dissolved at $60{ }^{\circ} \mathrm{C}$ in a $0.5 \%(w / v)$ aqueous chitosan solutions containing $1.0 \%(v / v)$ of acetic acid. The concentrations of agarose in the mixed solutions were 1.0, 1.5 and $2.0 \%(w / v)$. Then, mixed solutions were poured in Teflon molds and maintained overnight at $4{ }^{\circ} \mathrm{C}$ to allow the mixtures to gel. Blank hydrogels were designated as Chi/Aga-1, Chi/Aga-1.5 and Chi/Aga-2.

Chi/Aga ferrogels were prepared by mixing the ferrofluid solutions (with an initial concentration of $2.0 \%$ or $5.0 \%(w / v))$ with the corresponding Chi/Aga solutions under vortex agitation in a volume ratio of 6:1, until getting homogeneous samples. Then, mixed solutions were poured in Teflon molds and maintained overnight at $4{ }^{\circ} \mathrm{C}$ to allow the mixtures to gel. Ferrogels were designated as Chi/Aga+Fex, where $\mathrm{x}$ corresponds to the initial ferrofluid concentration, $2.0 \%$ or $5.0 \%(w / v)$.

The water content was determined by weighing the fully hydrated sample $(m \mathrm{~h})$ and the dried sample obtained by freeze-drying $\left(m_{0}\right)$ so that water content $(\%)=\left(m_{\mathrm{h}}-m_{0}\right) / m_{0}$.

\subsection{Scanning Electron Microscopy (SEM)}

The surface and cross section of all the samples under study were examined by scanning Electron Microscopy (SEM) (XL30 ESEM, Philips). All the gels under study were freeze-dried. Subsequently, the dried samples were coated with an ultrathin coating of gold deposited on the sample by high-vacuum metallization. Analysis of the pore structure was done using Image Pro 5.0 (media Cybernetics, Maryland, USA) software.

\subsection{Thermogravimetric Analysis}

The thermal stability of composite Chi/Aga macrogels was evaluated by thermogravimetric analysis (TGA) performed on a Q500 TA Instruments TGA, using a nitrogen stream as purge gas, at a heating rate of $10^{\circ} \mathrm{C} / \mathrm{min}$ in the range of temperature of $25-700{ }^{\circ} \mathrm{C}$. The experiments were carried out on freeze-dried samples.

\subsection{Dynamic Oscillatory Measurements}

Viscoelastic properties were determined in an AR-G2 rheometer (TA Instruments, Delaware, USA) using

$20 \mathrm{~mm}$-diameter steel parallel plates. Temperature sweeps were performed from 5 to $80{ }^{\circ} \mathrm{C}$ at $10^{\circ} \mathrm{C} / \mathrm{min}$ and at $1 \mathrm{~Hz}$ frequency. All the experiments were carried out at a fixed torque in the linear viscoelastic regime. The linear viscoelastic region was located with the aid of a torque sweep. All the viscoelastic measurements were performed on hydrogels swelled to equilibrium.

\subsection{Determination of Magnetic Remote Heating}

The specific power absorption (SPA) of Chi/Aga-1.5 ferrogels loaded with two concentrations of ferrofluid $(2.0 \%$ and $5.0 \% \mathrm{w} / \mathrm{v})$ was determined from measurements performed in a commercial $\mathrm{AC}$ field applicator (DM100 by $\mathrm{nB}$ nanoscale Biomagnetics, Spain) working at $f=418.5 \mathrm{kHz}$ and 
$24 \mathrm{kA} / \mathrm{m}(\approx 300 \mathrm{Oe})$. Experiments were carried out within a thermally-insulated working space of about $1 \mathrm{~cm}^{3}$, using a closed container of $1.0 \mathrm{~mL}$ volume. Samples for measurement were prepared directly in the vial.

\section{Acknowledgments}

Vanessa Zamora-Mora thanks CSIC for a JAE predoc fellowship. Rebeca Hernández acknowledges MINECO for a Ramon y Cajal fellowship. Financial support from MINECO (MAT 2011-24797 and MAT 2014-53437-C2-1) is gratefully acknowledged. Paula I.P. Soares and Coro Echeverria acknowledge support from the Portuguese Science and Technology Foundation through grants SFRH/BD/81711/2011, SFRH/BPD/88779/2012 and project UID/CTM/500025/2013.

\section{Author Contributions}

All authors of this paper have directly participated in the planning or drafting of this manuscript and have read and approved the final version submitted.

\section{Conflicts of Interest}

The authors declare no conflict of interest.

\section{References}

1. Buwalda, S.J.; Boere, K.W. M.; Dijkstra, P. J.; Feijen, J.; Vermonden, T.; Hennink, W. E. Hydrogels in a historical perspective: From simple networks to smart materials. J. Control. Release 2014, 190, 254-273.

2. Bhattarai, N.; Gunn, J.; Zhang, M. Chitosan-based hydrogels for controlled, localized drug delivery. Adv. Drug Deliv. Rev. 2010, 62, 83-99.

3. Dang, J.M.; Leong, K.W. Natural polymers for gene delivery and tissue engineering. Adv. Drug Deliv. Rev. 2006, 58, 487-499.

4. Majedi, F.S.; Hasani-Sadrabadi, M.M.; Emami, S.H.; Taghipoor, M.; Dashtimoghadam, E.; Bertsch, A.; Moaddel, H.; Renaud, P. Microfluidic synthesis of chitosan-based nanoparticles for fuel cell applications. Chem. Commun. 2012, 48, 7744-7746.

5. Muzzarelli, R.A.A.; Boudrant, J.; Meyer, D.; Manno, N.; DeMarchis, M.; Paoletti, M. G. Current views on fungal chitin/chitosan, human chitinases, food preservation, glucans, pectins and inulin: A tribute to Henri Braconnot, precursor of the carbohydrate polymers science, on the chitin bicentennial. Carbohyd. Polymer. 2012, 87, 995-1012.

6. Muzzarelli, R. A. A.; Muzzarelli, C. Chitosan Chemistry: Relevance to the Biomedical Sciences. In Polysaccharides I, Heinze, T., Ed. Springer Berlin Heidelberg: Berlin, Germany, 2005; 186, pp 151-209.

7. Dash, M.; Chiellini, F.; Ottenbrite, R. M.; Chiellini, E. Chitosan-A versatile semi-synthetic polymer in biomedical applications. Prog. Polymer Sci. 2011, 36, 981-1014.

8. Park, J. H.; Saravanakumar, G.; Kim, K.; Kwon, I. C. Targeted delivery of low molecular drugs using chitosan and its derivatives. Adv. Drug Deliv. Rev. 2010, 62, 28-41. 
9. Puga, A. M.; Lima, A. C.; Mano, J. F.; Concheiro, A.; Alvarez-Lorenzo, C. Pectin-coated chitosan microgels crosslinked on superhydrophobic surfaces for 5-fluorouracil encapsulation. Carbohyd. Polymer. 2013, 98, 331-340.

10. Zhang, H.; Mardyani, S.; Chan, W. C. W.; Kumacheva, E. Design of biocompatible chitosan microgels for targeted $\mathrm{pH}$-mediated intracellular release of cancer therapeutics. Biomacromolecules 2006, 7, 1568-1572.

11. Wei, L.; Cai, C.; Lin, J.; Wang, L.; Zhang, X. Degradation controllable biomaterials constructed from lysozyme-loaded Ca-alginate microparticle/chitosan composites. Polymer 2011, 52, 5139-5148.

12. Hernández, R.; Zamora-Mora, V.; Sibaja-Ballestero, M.; Vega-Baudrit, J.; López, D.; Mijangos, C. Influence of iron oxide nanoparticles on the rheological properties of hybrid chitosan ferrogels. J. Colloid Inter. Sci. 2009, 339, 53-59.

13. Hee Kim, E.; Sook Lee, H.; Kook Kwak, B.; Kim, B.-K. Synthesis of ferrofluid with magnetic nanoparticles by sonochemical method for MRI contrast agent. J. Magnetism Magnet. Mater. 2005, 289, 328-330.

14. Li, G.-Y.; Huang, K.-L.; Jiang, Y.-R.; Ding, P.; Yang, D.-L. Preparation and characterization of carboxyl functionalization of chitosan derivative magnetic nanoparticles. Biochem.Eng. J. 2008, 40, 408-414.

15. Hernandez, R.; Sacristan, J.; Nogales, A.; Fernandez, M.; Ezquerra, T. A.; Mijangos, C. Structure and viscoelastic properties of hybrid ferrogels with iron oxide nanoparticles synthesized in situ. Soft Matter 2010, 6, 3910-3917.

16. Li, Y.; Huang, G.; Zhang, X.; Li, B.; Chen, Y.; Lu, T.; Lu, T. J.; Xu, F. Magnetic hydrogels and their potential biomedical applications. Adv. Funct. Mater. 2013, 23 (6), 660-672.

17. Hernández, R.; Sacristán, J.; Asín, L.; Torres, T. E.; Ibarra, M. R.; Goya, G. F.; Mijangos, C. Magnetic hydrogels derived from polysaccharides with improved specific power absorption: Potential devices for remotely triggered drug delivery. J. Phys. Chem. B 2010, 114, 12002-12007.

18. Wang, L.; Stegemann, J. P. Thermogelling chitosan and collagen composite hydrogels initiated with $\beta$-glycerophosphate for bone tissue engineering. Biomaterials 2010, 31, 3976-3985.

19. Farshi Azhar, F.; Olad, A.; Salehi, R. Fabrication and characterization of chitosangelatin/nanohydroxyapatite-polyaniline composite with potential application in tissue engineering scaffolds. Des. Monomers Polym. 2014, 17, 654-667.

20. Miguel, S. P.; Ribeiro, M. P.; Brancal, H.; Coutinho, P.; Correia, I. J. Thermoresponsive chitosanagarose hydrogel for skin regeneration. Carbohyd. Polym. 2014, 111, 366-373.

21. Wang, N.; Wu, X. S. Preparation and characterization of agarose hydrogel nanoparticles for protein and peptide drug delivery. Pharm. Dev. Technol. 1997, 2, 135-142.

22. Buckley, C.T.; Thorpe, S.D.; O’Brien, F.J.; Robinson, A.J.; Kelly, D.J. The effect of concentration, thermal history and cell seeding density on the initial mechanical properties of agarose hydrogels. J. Mech. Behav. Biomed. Mater. 2009, 2, 512-521.

23. Cao, Z.; Gilbert, R. J.; He, W. Simple Agarose-Chitosan gel composite system for enhanced neuronal growth in three dimensions. Biomacromolecules 2009, 10, 2954-2959. 
24. Xue, Z.-X.; Yang, G.-P.; Wang, G.-C.; Niu, J.-F.; Cao, X.-Y. Preparation of porous chitosan/agarose microsphere and its R-phycoerythrin release properties. J. Appl. Polym. Sci. 2007, 103, 2759-2766.

25. Hu, Z.; Li, S.; Yang, L., Preparation of berbamine loaded chitosan-agarose microspheres and in vitro release study. Polímeros 2012, 22 (5), 422-426.

26. Teng, S.-H.; Wang, P.; Kim, H.-E. Blend fibers of chitosan-agarose by electrospinning. Mater. Let. 2009, 63, 2510-2512.

27. Zamora-Mora, V.; Velasco, D.; Hernández, R.; Mijangos, C.; Kumacheva, E. Chitosan/agarose hydrogels: Cooperative properties and microfluidic preparation. Carbohyd. Polym. 2014, 111, 348-355.

28. Peniche-Covas, C.; Argüelles-Monal, W.; San Román, J. A kinetic study of the thermal degradation of chitosan and a mercaptan derivative of chitosan. Polym. Degrad. Stabil. 1993, 39, $21-28$.

29. Zhang, L. M.; Wu, C. X.; Huang, J. Y.; Peng, X. H.; Chen, P.; Tang, S. Q. Synthesis and characterization of a degradable composite agarose/HA hydrogel. Carbohyd. Polym. 2012, 88, $1445-1452$.

30. Lima, E., Jr.; Torres, T. E.; Rossi, L. M.; Rechenberg, H. R.; Berquo, T. S.; Ibarra, A.; Marquina, C.; Ibarra, M. R.; Goya, G. F. Size dependence of the magnetic relaxation and specific power absorption in iron oxide nanoparticles. J. Nanopart. Res. 2013, 15, 1-11.

31. Kumar, C. S. S. R.; Mohammad, F. Magnetic nanomaterials for hyperthermia-based therapy and controlled drug delivery. Adv. Drug Deliv. Rev. 2011, 63, 789-808.

32. Sadat, M. E.; Patel, R.; Sookoor, J.; Bud'ko, S. L.; Ewing, R. C.; Zhang, J.; Xu, H.; Wang, Y.; Pauletti, G. M.; Mast, D. B.; Shi, D. Effect of spatial confinement on magnetic hyperthermia via dipolar interactions in $\mathrm{Fe}_{3} \mathrm{O}_{4}$ nanoparticles for biomedical applications. Mater. Sci. Eng.: C Mater. Biol Appl. 2014, 42, 52-63.

(C) 2015 by the authors; licensee MDPI, Basel, Switzerland. This article is an open access article distributed under the terms and conditions of the Creative Commons Attribution license (http://creativecommons.org/licenses/by/4.0/). 